\title{
On-axis injection scheme based on a triple-frequency rf system for diffraction-limited storage rings
}

\author{
Shichang Jiang ${ }^{*}$ and Gang Xu \\ Institute of High Energy Physics, CAS, the University of Chinese Academy of Sciences, \\ Beijing 100049, People's Republic of China
}

(Received 7 August 2018; published 2 November 2018)

\begin{abstract}
As the multibend achromats had been applied to the lattice design in latest light sources to achieve ultralow beam emittance, whereas its performance is restricted by the strong sextupoles and concomitant nonlinearities to a certain extent. Contractive dynamic aperture becomes an inevitable but pivotal characteristic of future diffraction-limited storage rings, which lead to the exclusion of the conventional off-axis injection schemes. In this paper we will present a new on-axis injection scheme in allusion to future light sources, which is based on a triple-frequency rf system. By means of delicate superposition of $\mathrm{rf}$ voltages with fundamental, 2nd and 3rd hamonic frequencies, a commodious and rational main bucket which can accommodate an injected bunch and a circulating bunch simultaneously is able to be formed. The injected bunch can be captured by the longitudinal acceptance on-axis in a reasonable time offset with respect to the circulating bunch, utilizing the ultrafast pulse kicker, and ultimately merging to the center of the main bucket through synchrotron radiation damping. Detailed construction of the triple-frequency rf system and application of this scheme to the High Energy Photon Source will be discussed in the paper, relevant simulation studies and discussions are also presented.
\end{abstract}

DOI: 10.1103/PhysRevAccelBeams.21.110701

\section{INTRODUCTION}

Along with the increasing newly constructions and upgrade projects of the light source worldwide, it has been become a strong embranchment in the accelerator field, meanwhile driving the development of corresponding physics and technologies. To achieve the ultimate target in this vigorous field, which is alleged diffraction-limited storage ring (DLSR) [1], the innovative multibend achromat (MBA) lattice design [2] and its variants have turned into the requisite approach to beat this target nowadays. Through the combination of several dipoles in a suitable length to bend electrons, high gradient quadrupoles to make strong focusing and dedispersion, and several arranged subtly sets of sextupoles to compensate negative chromaticities, one can assemble easily a standard achromat section. After that choosing legitimate periodicity to splice all these achromat sections, combining the beam injection and other functional sections, one preliminary layout of the modern light source is produced. Ineluctable consequences to this design is that the dynamic aperture begins shrinking dramatically while optimizing a beam

\footnotetext{
*jiangsc@ihep.ac.cn
}

Published by the American Physical Society under the terms of the Creative Commons Attribution 4.0 International license. Further distribution of this work must maintain attribution to the author(s) and the published article's title, journal citation, and DOI. emittance towards the diffraction-limited, corresponding value in modern light sources is nearly one-tenth compared to the machines in the last century. How to make the tradeoff in beam emittance and dynamic aperture as well as the other nonlinear effects is a principle issue in the lattice design.

Common understanding to the dramatic shrinking of the dynamic aperture is the exclusion of the conventional off-axis injection schemes, which are usually constituted of bump kickers and septum magnets [3]. A local orbit bump in the injection period is produced by a set of bump kickers, to enable the circulating bunches can be closer to the injected bunch, and they are separated in the landscape orientation. This scheme usually demands sufficient dynamic aperture of the storage ring, which should be larger than the total separation between the circulating bunches with respect to the injected bunch, and the thickness of the septum. Evidently the dynamic aperture within a few millimeters cannot be compatible with such injection scheme. Beyond that during continual beam injection on the top-up mode, this scheme still makes certain disturbances to the circulating bunches due to the bumped orbit.

In order to satisfy the injection requirements of modern light sources, several novel and inventive injection schemes have been proposed in recent years. Multipole kicker injection scheme had been studied $[4,5]$ and designed in MAX IV [6], although it is still an off-axis injection scheme, the restriction to the dynamic aperture is much looser compared to the former scheme using the bump kickers. 
Through delicately designed the multipole magnet as kicker, quad or higher multipole, which enables the circulating bunches pass through the center of the kicker without disturbance, the injected bunch with a horizontal offset can be kicked appropriately by a special nonlinear pulse waveform, and captured by the horizontal acceptance finally.

Apart from these off-axis injection schemes, more efficient approaches to relax the requirement to the dynamic aperture are the on-axis injection schemes. The longitudinal injection scheme using a short pulse kicker proposed by M. Aiba had been a great pointcut and living example for the subsequent designs [7]. By injecting an electron bunch onto the close orbit with a time offset to the circulating bunch, utilizing a fast pulse dipole kicker, the injected bunch can be merged to the main bucket through synchrotron radiation damping. Besides it can be injected into the bucket with a designed energy deviation and time offset with respect to the circulating bunch, being described as the "golf club" effect. This injection scheme tactfully transfers the pressure from the severe restriction of the dynamic aperture to the pulse kicker design.

The another class of on-axis injection schemes, as being adopted by the majority of the latest light sources, is the swap-out injection scheme [8-10]. The circulating bunch will be completely replaced by the injected bunch using a pulse kicker in a designed time sequence, one can also choose to replace a long bunch train rather than a single bunch. This design relaxes the restriction to the dynamic aperture, and the technical requirements to the fast pulse kicker, which owes better practicability compared to the other on-axis injection schemes. However the particular considerations to the injector in this scheme are necessary, since the circulating bunches can only be replaced by the bunches from the injector. The stable storage to these high charged bunches in the injector and how to deal with the dumped bunches from the storage ring are still worth considering prudently.

A double-frequency rf system utilized for the injection scheme in the storage ring can be traced back to the Novosibirsk positron storage ring VEPP-3 [11], and the similar injection schemes are able to achieved in the light sources theoretically. These schemes aim to produce a fresh bucket to accommodate the injected bunch with a time offset with respect to the original bucket, which is alleged if gymnastics. B.C Jiang et al. utilize the fundamental and 3rd harmonic cavities, by altering rf voltages in the injection period to make it [12]. Once the injected bunch is captured by the fresh bucket, the inverse process will make sure these two buckets are able to fuse one main bucket in the normal operation, as well as the bunches. Gang Xu et al. also achieve similar process [13], by means of adjusting both rf voltages and phases. These designs only propose the rigorous demand to the ultrafast pulse kicker, particularly the raise time of the pulse, and also relax the restriction to the dynamic aperture. Nevertheless continual injection in the top-up mode means continual adjustments to the $\mathrm{rf}$ parameters, which is a severe challenge to rf hardwares and a control system. The original bucket during such injection process may be deformed, which leads to the shrink of the bunch length.

Using two kinds of nonlinear kickers (NLK) to achieve a novel longitudinal injection, including a transverse NLK without constraint in duration and a longitudinal NLK to improve the capture of a off-momentum beam, is proposed by Marie-Agnès Tordeux [14]. The scheme evades the usage of a ultrafast pulse kicker and the swap-out method. Through the combination of the fundamental and 3rd harmonic cavities, one can keep the stored bunches unaffected which stay at the synchrotron phase, and reduce the momentum deviation of the injected bunch as soon as possible. The dynamic aperture under a large momentum deviation with a time offset respecting to a synchrotron phase is pivotal in this scheme, in which the lattice may have to be optimized specifically, to avoid the injected bunch getting lost initially.

In this paper we will propose a new on-axis injection scheme for future diffraction-limited storage rings inspired by these aforementioned remarkable schemes, which is based on a triple-frequency rf system. By means of delicate superposition of rf voltages with fundamental, 2nd, and 3rd harmonic frequencies, a commodious and rational main bucket is able to be formed, which can accommodate the injected bunch and circulating bunch simultaneously. rf gymnastics in a double-frequency rf system is not necessary any more. While the 3rd harmonic cavity can help prolong the bunch length to relief the emittance growth caused by the IBS effect. The injected bunch is transferred to the injection point with a time offset to the circulating bunch, being kicked and captured by the longitudinal acceptance in a designed time sequence, while the circulating bunch still stays at the synchrotron phase without disturbance. The time interval between the outermost injection point and the circulating bunch is larger than the raise time of the kicker pulse, hence this process is nearly transparent to the user experiments. And the whole injection process can be done in multiturn, thereby relaxing the requirements to the injector while operating on the high charge mode. Above all, the entire rf parameters will remain unchanged in the normal operation.

We apply the scheme to the one lattice of the High Energy Photon Source (HEPS) particularly, in Sec. II we will describe the injection requirements of HEPS first. The construction and filtration to the rf parameters of the above triple-frequency rf system will be discussed in Sec. III. Relevant simulation studies will be presented in Sec. IV. Especially when considering the energy loss per turn related to the momentum deviation, as well as alleged "golf club" effect, the time interval between the outermost injection point and the circulating bunch can be further prolonged. And we will discuss it in Sec. V. The final conclusion and other discussions will be given in Sec. VI. 
TABLE I. Major parameters of HEPS, noted that energy loss per turn and relevant parameters by 15 insert devices are included, values are derived from ELEGANT simulation.

\begin{tabular}{lccc}
\hline \hline Parameters & Symbols & Values & Units \\
\hline Circumference & $C$ & 1360.4 & $\mathrm{~m}$ \\
Beam energy & $E_{0}$ & 6 & $\mathrm{GeV}$ \\
Beam current & $I_{0}$ & 200 & $\mathrm{~mA}$ \\
Natural emittance & $\epsilon_{0}$ & 26.14 & $\mathrm{pm}$ \\
Betatron tunes & $\nu_{x} / \nu_{y}$ & $114.19 / 106.18$ & \\
Momentum compaction & $\alpha_{c}$ & $1.28 \mathrm{e}-5$ & \\
Natural energy spread & $\delta_{p} / \mathrm{p}$ & $1.14 \mathrm{e}-3$ & \\
Energy loss per turn & $U_{0}$ & 4.38 & $\mathrm{MeV}$ \\
Harmonic number & $h_{0}$ & 756 & \\
\hline \hline
\end{tabular}

\section{THE INJECTION REQUIREMENTS OF HEPS}

High Energy Photon Source (HEPS) is a newly designed $6 \mathrm{GeV}$ light source [15], its major parameters are listed in Table I, which will be used for mathematical analysis and simulations in the next sections. Corresponding beta and dispersion functions in the standard achromat section are presented in Fig. 1.

According to the previous physical design to the injection scheme, the harmonic number is 756 and a corresponding frequency of the fundamental rf cavity is 166.6 $\mathrm{MHz}$, indicating the time interval between an electron bunch in a uniform fill pattern is $6 \mathrm{~ns}$, which is the time boundary for the longitudinal injection scheme. Subjecting to the practical technological limitation, certain raise time for a ultrafast pulse kicker is indispensable, whose a limiting value under current state of art is around 2 ns. The strip-line type kicker and its associated power supply design is the leading-edge technology in a relevant field nowadays, and being developed in several light sources $[16,17]$. Thus the outermost injection point in our scheme must be far from the circulating bunch with a time offset around $2 \mathrm{~ns}$, otherwise there is no sufficient time to raise a full strength pulse, or disturbing the circulating bunch if one wants to bring forward the raising of this
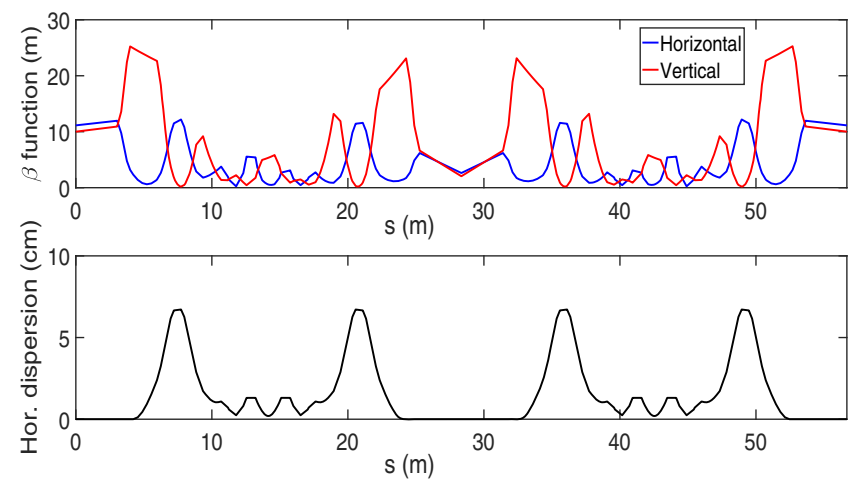

FIG. 1. Lattice function in the standard achromat section of the HEPS $6 \mathrm{GeV}$ storage ring, which is composed of 24 such similar cells.

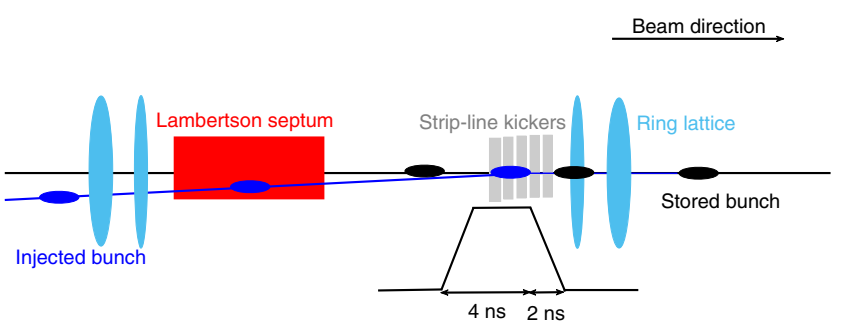

FIG. 2. Schematic diagram of a uniform filling pattern of HEPS, the circles filling with black present the circulating bunches in the storage ring, while the circles filling with blue stands for the injected bunch. A time interval between the circulating bunches is $6 \mathrm{~ns}$.

pulse. Figure 2 shows a simplified sequence chart of the above narration.

The reason why the foregoing on-axis injection scheme based on a double-frequency rf system utilizing intricate rf gymnastics is here. A more straightforward idea is to form one main bucket at first, which satisfies both our injection requirements and the limitation of the ultrafast pulse kicker. Now we start to construct such special main bucket.

\section{CONSTRUCTION OF THE TRIPLE-FREQUENCY RF SYSTEM}

While utilizing such complex rf system to inject the bunch, meticulous selection to the rf parameters is our first step. We will describe our approaches to make it in this section, meanwhile noted that this is not the only way, just a choice of mathematical treatments.

Without synchrotron radiation, the longitudinal motion of the particles in a triple-frequency rf system can be described by Hamiltonian:

$$
\begin{aligned}
H(\phi, \delta ; t)= & \frac{h_{f} \omega_{0} \eta}{2} \delta^{2}+\frac{\omega_{0} e}{2 \pi E_{0} \beta^{2}}\left[\sum_{i=1}^{N_{f}} V_{f}^{i} \cos \left(\phi+\phi_{f}^{i}\right)\right. \\
& +\frac{h_{f}}{h_{1}} \sum_{j=1}^{N_{h_{1}}} V_{h_{1}}^{j} \cos \left(\frac{h_{1}}{h_{f}} \phi+\phi_{h_{1}}^{j}\right) \\
& \left.+\frac{h_{f}}{h_{2}} \sum_{k=1}^{N_{h_{2}}} V_{h_{2}}^{k} \cos \left(\frac{h_{2}}{h_{f}} \phi+\phi_{h_{2}}^{k}\right)+\phi U_{0}\right] .
\end{aligned}
$$

Where $\phi$ and $\delta$ are a pair canonical variables with respect to the time variable $t, \omega_{0}=2 \pi c / C$ is the angular revolution frequency of the synchrotron particle, $c$ is the speed of light, $C$ is the circumference of the storage ring. $\eta=\alpha_{c}-1 / \gamma^{2}, \beta=\sqrt{1-\gamma^{2}}$, where $\alpha_{c}$ is the momentum compaction factor of the storage ring, $\gamma$ is the relativistic factor. Suppose there are $N_{f}$ fundamental cavities with a harmonic number $h_{f}, N_{h_{1}}$ harmonic cavities with a harmonic number $h_{1}$, and $N_{h_{2}}$ harmonic cavities with a harmonic number $h_{2} . V_{f}^{i}, V_{h_{1}}^{j}$, and $V_{h_{2}}^{k}$ are the voltages of the $i$ th fundamental cavity, the $j$ th 2 nd harmonic cavity, 
and the $k$ th 3 rd harmonic cavity respectively. $\phi_{f}^{i}, \phi_{h_{1}}^{j}$, and $\phi_{h_{2}}^{k}$ are the phases of the synchrotron particle relative to the above cavities. To simplify the treatment, we choosing six independents rf parameters $V_{f}, V_{h_{1}}, V_{h_{2}}$ and $\phi_{f}, \phi_{h_{1}}, \phi_{h_{2}}$, which can be defined from the above relations:

$$
\begin{gathered}
V_{f} \cos \left(\phi+\phi_{f}\right)=\sum_{i=1}^{N_{f}} V_{f}^{i} \cos \left(\phi+\phi_{f}^{i}\right), \\
V_{h_{1}} \cos \left(\frac{h_{1}}{h_{f}} \phi+\phi_{h_{1}}\right)=\sum_{j=1}^{N_{h_{1}}} V_{h_{1}}^{j} \cos \left(\frac{h_{1}}{h_{f}} \phi+\phi_{h_{1}}^{j}\right), \\
V_{h_{2}} \cos \left(\frac{h_{2}}{h_{f}} \phi+\phi_{h_{2}}\right)=\sum_{k=1}^{N_{h_{2}}} V_{h_{2}}^{k} \cos \left(\frac{h_{2}}{h_{f}} \phi+\phi_{h_{2}}^{k}\right) .
\end{gathered}
$$

We can derive the expression of a potential energy function from Hamiltonian as

$$
\begin{aligned}
P(\phi ; t)= & U_{0}\left(\phi-\phi_{s}\right)+V_{f}\left[\cos \left(\phi+\phi_{f}\right)-\cos \left(\phi_{s}+\phi_{f}\right)\right] \\
& +\frac{V_{h_{1}}}{2}\left[\cos \left(2 \phi+\phi_{h_{1}}\right)-\cos \left(2 \phi_{s}+\phi_{h_{1}}\right)\right] \\
& +\frac{V_{h_{2}}}{3}\left[\cos \left(3 \phi+\phi_{h_{2}}\right)-\cos \left(3 \phi_{s}+\phi_{h_{2}}\right)\right] .
\end{aligned}
$$

Mathematically we add another variable in the next analysis, which is the fixed point of the longitudinal acceptance noted as $\phi_{b}$, and it can be described as

$$
P\left(\phi_{b}\right)=P_{\max }, \quad P^{\prime}\left(\phi_{b}\right)=0,
$$

where $P_{\max }=\delta_{B}^{2} \Phi, \Phi=\pi h_{f} \eta E_{0} \beta^{2}$, and $\delta_{B}$ is the bucket height.

To satisfy the requirements of the injection scheme, the function of the potential energy needs to be restricted with several conditions:

$$
\left\{\begin{array}{l}
P\left(\phi_{s}\right)=0 \\
P^{\prime}\left(\phi_{s}\right)=0, \\
P\left(\phi_{b}\right)=P_{\max } \\
P^{\prime}\left(\phi_{b}\right)=0, \\
\phi_{s}-\phi_{b} \approx 2 n s .
\end{array}\right.
$$

To satisfy the optimal bunch lengthening condition, there is another limitation:

$$
P^{\prime \prime}\left(\phi_{s}\right)=0 .
$$

Through solving these equations, the number of independent variables can be reduced, which can relive a lot stress from searching for the optimal rf parameters. We present a feasible approach to make it and the final solutions in Appendix, here no more detailed explanations. Table II presents one set of the solutions according to the aforementioned HEPS lattice. rf voltages, phases and corresponding beam powers are given respectively.
TABLE II. One set of the triple-frequency rf system parameters, noted that negative voltages of the fundamental and 2 nd harmonic cavity is due to our definition to a synchrotron phase is $\pi$, rather than 0 . The total beam power is $I_{0} U_{0}=876.279 \mathrm{~kW}$ with a designed average current $200 \mathrm{~mA}$.

\begin{tabular}{lccc}
\hline \hline Parameters & Fundamental & 2nd Harmonic & 3rd Harmonic \\
\hline Frequency & $166.6 \mathrm{MHz}$ & $333.2 \mathrm{MHz}$ & $499.8 \mathrm{MHz}$ \\
Voltage & $-7.16 \mathrm{MV}$ & $-3.59 \mathrm{MV}$ & $0.90 \mathrm{MV}$ \\
Phase & $2.41 \mathrm{rad}$ & $3.21 \mathrm{rad}$ & $0.82 \mathrm{rad}$ \\
Beam power & $957.88 \mathrm{~kW}$ & $50.10 \mathrm{~kW}$ & $-131.71 \mathrm{~kW}$ \\
\hline \hline
\end{tabular}

It is observed that the fundamental cavity supplies a large proportion of power to the beam, the 3rd harmonic cavity absorbs power from the beam while prolonging the bunch length, which conform to our common sense. There is little power exchange with the beam in the 2nd harmonic cavity, since their phase in our solutions are all around 0 degree, which just exerts an influence on lengthening the main bucket compared to a conventional bunch lengthening rf system. Even in several particular solutions, this value is exactly 0 degree.

Figure 3 presents the longitudinal acceptance, the function of potential energy and its corresponding first derivative, vary with horizontal axis phase $\phi$, using the above rf parameters. Noted that the bucket height is $3 \%$, the black star is the fixed point $\phi_{b}$, while the black hexagram is the synchrotron phase $\phi_{s}$, and the injection points can be
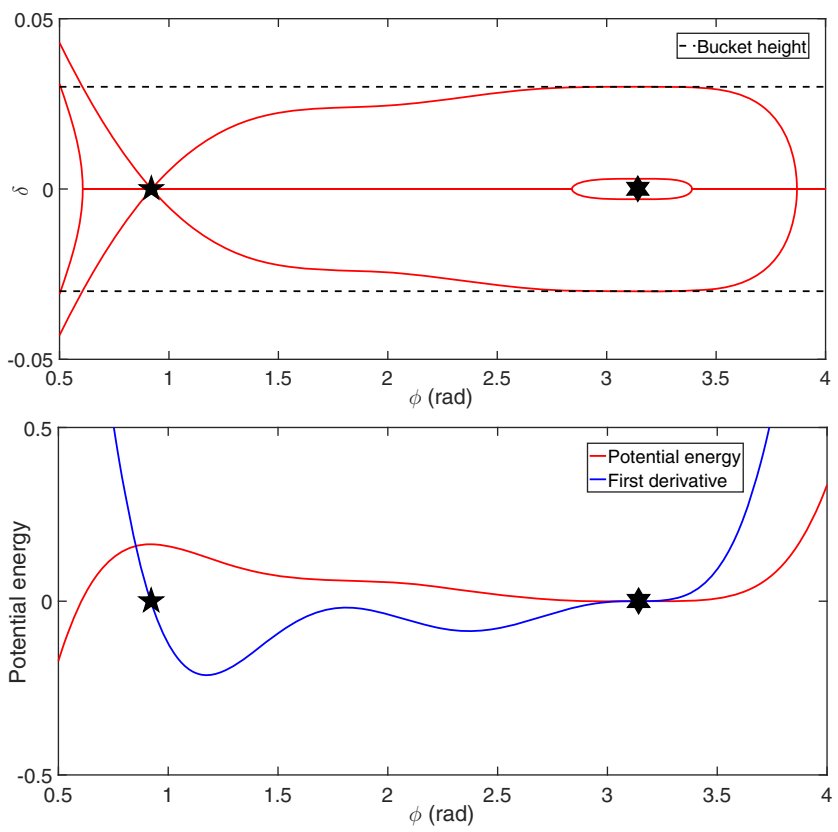

FIG. 3. Longitudinal acceptance, and potential energy, corresponding to the first derivative curve to be conducted using the above triple-frequency rf parameters. The black star presents the fixed point $\phi_{b}$, and the black hexagram stands for the synchrotron phase $\phi_{s}$. The different curves in the upper figure present the distinct energy deviation corresponding to $3 \%$ and a triple rms energy spread. 


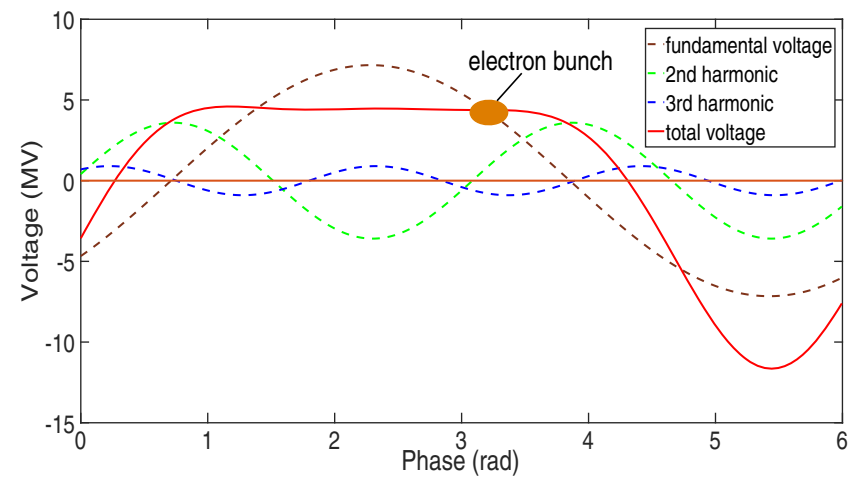

FIG. 4. rf voltage seen by the bunch formed by the triplefrequency rf system.

selected from the synchrotron phase $\phi_{s}$, to $\phi_{b}$. The rational time interval between them is decided by the raise time of the ultra-fast pulse kicker, avoiding affecting the circulating bunch, which is related to the practical technological level.

From our longitudinal acceptance in Fig. 3, the time interval between $\phi_{b}$ and the area where the circulating bunch stays, represented by the inner red ellipse in Fig. 3, is nearly $2 \mathrm{~ns}$, which meets our requirements basically. Figure 4 presents the synthetic rf voltage formed by such triple-frequency rf system, the circulating bunch stays at the synchrotron phase. The total voltage curve is quite flat around the synchrotron phase.

For easier comparison, we put the longitudinal acceptance and corresponding function of potential energy derived from only the one fundamental frequency cavity, the doublefrequency rf system, which is composed of the fundamental and 3rd harmonic cavities, and the triple-frequency rf system together in Fig. 5. Noted that the longitudinal scale of the primary bucket under the double-frequency rf system is somewhat larger than the single fundamental frequency, but still far away from $2 \mathrm{~ns}$, which is our essential criterion for the longitudinal injection scheme. Also this is our mentioned reason why the researchers painstakingly try novel tricks on a double-frequency rf system, since there is no sufficient time to raise a full strength pulse. No doubt that the adjunction of another 2 nd harmonic cavity in the original rf system composed of the fundamental and 3rd harmonic cavity can prolong the original main bucket, till satisfying our injection requirements.

If we apply the above triple-frequency rf system to the storage ring theoretically, the corresponding appropriate main bucket can be formed. During the injection period, once a circulating bunch transits the injection point, a trigger signal is sent to the power supply of the kicker. After nearly $2 \mathrm{~ns}$, the full flat-top pulse can be raised, while an injected bunch is transferred to the injection point exactly and kicked by this pulse, then it will be captured by the longitudinal acceptance. From then the pulse starts falling, and waiting for another injection. The injected bunch will merge to the centre of main bucket through longitudinal motion including synchrotron radiation damping, till now
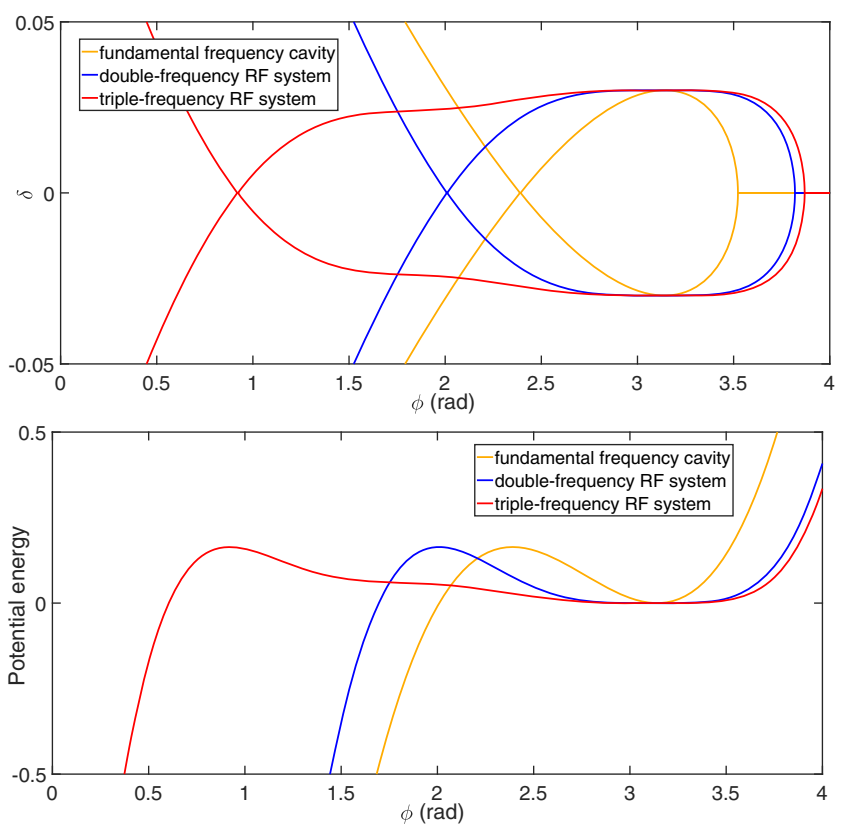

FIG. 5. Various longitudinal acceptances and potential energy curves under the different rf systems. Only the fundamental cavity, the double-frequency rf system and the triple-frequency rf system correspond to yellow, blue and red curves, respectively.

we finish a whole beam accumulation process. Similar process can be repeated until replenishing the average beam current to the designed value.

Compared to the before-mentioned on-axis beam accumulation scheme based on a double-frequency rf system, the biggest advantage is all our of parameters can remain unchanged. Without the need of rf gymnastics, we can let the 2nd harmonic and the 3rd harmonic cavities work on the passive mode, which relief the stress from the design of the rf cavities, as well as the low-level control system.

However the additional 2nd harmonic cavities mean extra input in a practical project, no matter the initial investment or routine maintenance. And the additional straight sections are needed to install them, which may be a problem in modern light sources, as the quite compact design is being adopted by most of them. More rf cavities mean more impedance elements in a storage ring, corresponding collective instabilities are still under study. Besides the rf noises may lead to the deformation of such potential curve, since it is quite flat around the synchrotron phase. And it might cause the shift of bunch centroid longitudinally. Various instabilities in such injection scheme will be our priority of work in the future.

\section{RELEVANT SIMULATION STUDIES USING THE ABOVE TRIPLE-FREQUENCY RF SYSTEM IN ELEGANT}

We utilize macro particles tracking code ELEGANT to simulate our injection process [18], based on the abovementioned HEPS lattice. ILMATRIX is utilized to make 
TABLE III. Parameters of the injected beam used in the following ELEGANT simulation.

\begin{tabular}{lc}
\hline \hline Parameters & Value \\
\hline Beam energy & $6 \mathrm{GeV}$ \\
RMS energy spread & $1 \mathrm{e}-3$ \\
Bunch length & $4 \mathrm{~mm}$ \\
Horizontal emittance & $4 \mathrm{~nm}$ \\
Vertical emittance & $2 \mathrm{~nm}$ \\
\hline \hline
\end{tabular}

single-turn beam transport, noted that the transverse nonlinearities are not included in it here, SREFFECTS provides the lumped simulation of synchrotron radiation. RFCA and RFMODE handle with the beam cavity interaction in ELEGANT respectively. The former one handles with the rf cavity as the first-order matrix, while the other one simulates the beam-driven part in the cavity using fundamental theorem of beam loading and phasor rotation [19].

At first we adopt RFCA in the simulation as a proof-ofprinciple process. Table III presents the major parameters of the injected bunch, which are assumed from the initial booster design. The triple-frequency rf system is built using RFCA with rf voltages and phases described in Table II.

Error studies are not included here, hence a bare HEPS lattice is used for tracking, including 15 insert devices defined by UKICKMAP in the lattice file. Figure 6 presents the longitudinal parameters of the injected bunch vary with the number of passes, the energy spread and bunch length are nearly the same as the initial numerical calculation, and the beam is indeed prolonged to nearly $3 \mathrm{~cm}$.

Particle distribution in the longitudinal plane is presented at Fig. 7, where the bunch is injected at $\mathrm{dt}=-2 \mathrm{~ns}$, $\mathrm{dp}=0$, and stays up to over 40000 turns. The longitudinal damping time is around $9 \mathrm{~ms}$, corresponding to 2000 turns. Once the injected bunch starts merging to the center of the main bucket through longitudinal motion, the longitudinal distribution also becomes dispersive, since our designed 3rd harmonic cavity help prolong the bunch.

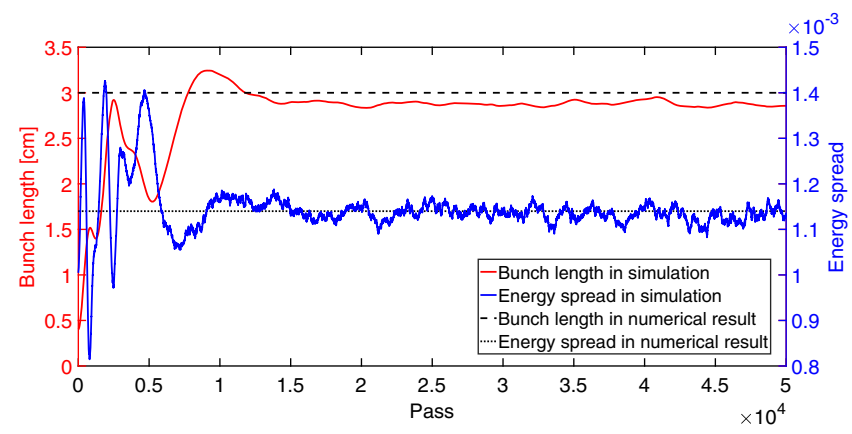

FIG. 6. Beam longitudinal parameters vary with the number of passes up to over 40000 turns, the red line stands for bunch lengths, while the blue one is energy spreads. The first dashed is the final bunch length derived from the bunch distribution numerically, and the second dashed is energy spreads calculated from the radiation integrals.

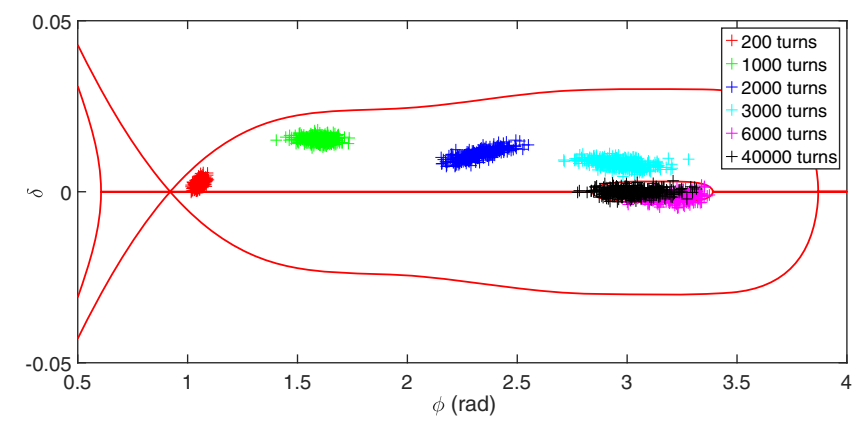

FIG. 7. Particle distribution in the longitudinal plane with up to 40000 turns, the bunch is injected at $\mathrm{dt}=-2 \mathrm{~ns}, \mathrm{dp}=0$.

The whole injection process is done within the longitudinal acceptance, and no particle loss during this period. Thus we testify the validity of our theory through RFCA, the injection scheme can indeed work in the theory, and bunch lengthening condition is also able to be satisfied.

As a matter of fact, once utilizing such complex rf system in an injection scheme, beam loading effect has to be brought to the forefront. The interaction of the beam with the cavity can be described mathematically, by the impedance of the cavity fundamental mode [20,21],

$$
Z(\omega)=\frac{R_{s}}{1+i Q\left(\frac{\omega_{r}}{\omega}-\frac{\omega}{\omega_{r}}\right)}=R_{s} e^{-i \Psi} \cos \Psi,
$$

where $R_{s}$ is the shunt impedance, $Q$ is the quality factor, $\omega_{r}$ is the resonant frequency for the cavity. The detuning angle of the cavity can be defined as $\tan \Psi=Q\left(\frac{\omega_{r}}{\omega}-\frac{\omega}{\omega_{r}}\right) \approx$ $2 Q \frac{\omega_{r}-\omega}{\omega_{r}}$. The beam-induced voltage on the cavity can be written as

$$
V(\tau)=-2 I_{0} R_{S} F \cos \Psi \cos \left(\omega_{h} \tau+\Psi-\Phi_{F}\right),
$$

where the bunch form factor is $F$ and its related phase $\Phi_{F}$, and $\omega_{h}$ is the angular frequency of the harmonic cavity.

The steady-state field is able to be generated through a certain filling time, which can be estimated through the rf hardware parameters as $T_{f}=\frac{2 Q}{\omega}$. And the field in the cavity is related to the filling pattern in the storage ring, when there is the large gap between the bunches or bunch trains, transient beam loading is dominant, and it may compromise the ability to control the beam. At certain circumstance it can be estimated by [22]

$$
\Delta \phi \approx \frac{I_{0}}{2 V_{f}} \frac{R_{s}}{Q} \omega_{r f} \Delta t
$$

where $\Delta \phi$ is the phase shift between the head and tail bunches, $\Delta t$ is the time interval between bunches. This formula is effective when $T_{0} \ll T_{f}$, in which $T_{0}$ is the revolution period.

While taking beam cavity interaction into account, the rf cavity can be divided into active and passive. Within the passive cavity, the electromagnetic field is completely driven 
TABLE IV. Hardware parameters of the triple-frequency $\mathrm{rf}$ system using in simulation, the 2nd and 3rd harmonic cavities are passive, while the main cavities work on active.

\begin{tabular}{lccc}
\hline \hline Parameters & Funda. & 2nd Harm. & 3rd Harm. \\
\hline Frequency & $166.6 \mathrm{MHz}$ & $333.2 \mathrm{MHz}$ & $499.8 \mathrm{MHz}$ \\
Voltage & $7.16 \mathrm{MV}$ & $\backslash$ & $\backslash$ \\
Phase & $2.34 \mathrm{rad}$ & $\backslash$ & $\backslash$ \\
Quality factor & $5 \mathrm{e} 8$ & $6 \mathrm{e} 8$ & $1 \mathrm{e} 9$ \\
Shunt impedance & $33950 \mathrm{M} \Omega$ & $36000 \mathrm{M} \Omega$ & $46750 \mathrm{M} \Omega$ \\
Detuning frequency & $-0.22 \mathrm{kHz}$ & $1.26 \mathrm{kHz}$ & $-4.35 \mathrm{kHz}$ \\
\hline \hline
\end{tabular}

by beam itself, and decelerate the beam. As the field of an active one is generated from the generator outside, the cavity voltage and phase are controllable to some extent. It's indispensable under several specific conditions, such as the before-mentioned on-axis injection scheme utilizing $\mathrm{rf}$ gymnastics, one have to adjust the rf voltage or phase manually. However the passive cavity is stabler, if the beam is stored steady in the storage ring. It is also a mainstream choice nowadays while the harmonic cavity is utilized in the latest light sources to prolong the bunch length.

RFMODE is taken to simulate the beam-driven part in ELEGANT, which had been exploited and adopted for the APS-U primary design [23], and a realistic model of rf feedback is included in RFMODE [19]. In our subsequent simulation, we adopt the active cavities as the fundamental frequency, and the other harmonic cavities are passive, all of them are working on the superconducting condition. Relevant major hardware parameters are presented at Table IV, initial beam parameters are the same as Table III. Noted that till now we just consider the uniform filling pattern, from the empirical formula Eq. (10), the phase shift between the head and tail bunch is less than $1 \times 10^{-3} \mathrm{rad}$, transient beam loading is secondary under such circumstance. RFMODE is utilized in the lattice file to establish our triple-frequency rf system.

Relevant simulation results of all three different types of cavities are shown at Fig. 8. The upper figure presents the longitudinal parameters vary with the number of the passes, the bunch length is indeed prolonged, which is decided by the detuning frequencies of the 2 nd harmonic and 3rd harmonic cavities. The bunch lengthening condition is still satisfied including the beam cavity interaction. The cavity voltage has certain concussion along with the establishment of the field driven by the beam. After over 9000 turns, when the beam-driven field reaches steady-state, the fundamental cavity voltage tends to be gentle, the cavity phase undergoes the similar process. The beam-induced voltages in the 2nd harmonic and 3rd harmonic cavities can also reach the target values in Table II, along with the average current up to the designed value $200 \mathrm{~mA}$.

Due to we utilize the 2nd harmonic and 3rd harmonic passive cavities, their detuning frequencies are actually two independent variables, corresponding to a phase in a
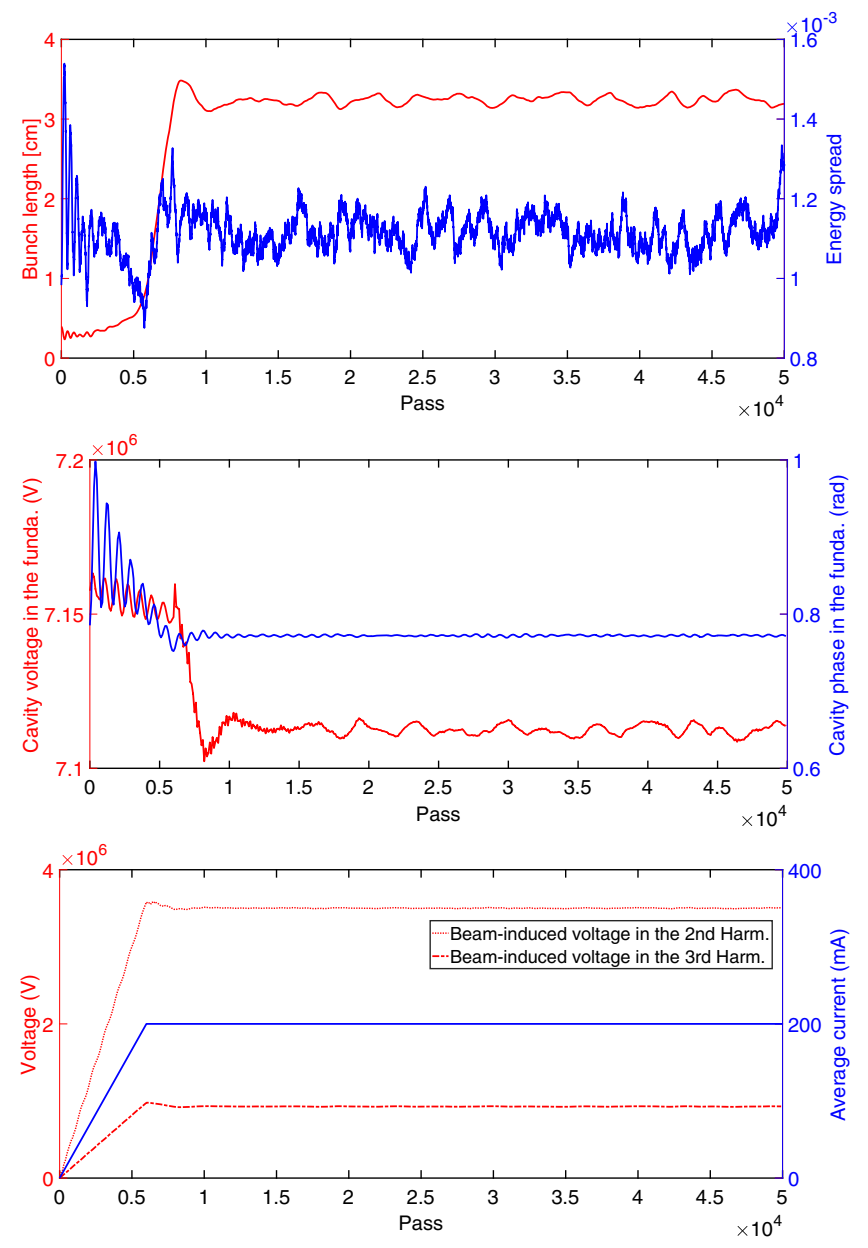

FIG. 8. The upper figure presents the longitudinal parameters of the beam vary with the number of passes, where the red line is the bunch length and the blue one is the energy spread. The center figure shows the cavity voltage and cavity phase of the main cavity along with the beam accumulated respectively. The bottom figure presents the beam-induced voltage in the 2 nd hamonic and 3rd harmonic passive cavities, while the blue line stands for the average current in the storage ring.

passive cavity, as $n \phi_{h}=\frac{\pi}{2}-\Psi$. Then we do the planar scanning to such two variables. Figure 9 shows the preliminary result using RFMODE, the colorbar from the dark blue to the dark red represents the bunch length from short to long, which is derived at the final steady-state in the simulation. The bunch length distribution has certain regularity, in some areas the bunch is precisely prolonged, while it will be over-stretched if the detuning frequency exceed certain range.

At Fig. 10 we pick four typical areas in the above result to record their final bunch distribution using HISTOGRAM in ELEGANT. Noted that the bunch is overstretched in the area 3 and area 4, as there are actually two local high spots on the longitudinal distribution.

Till now we present the relevant simulation studies using both RFCA and RFMODE. However we just utilize the bare lattice without any random errors on the lattice elements, 


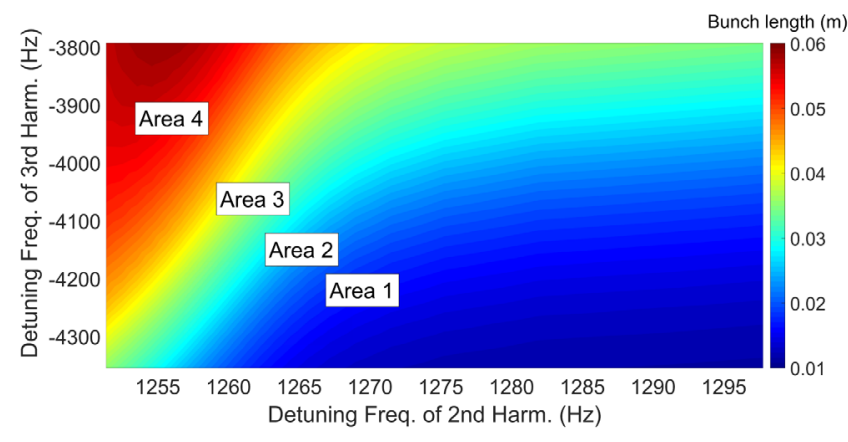

FIG. 9. Bunch length distribution varies with the detuning frequency of the 2nd and 3rd harmonic cavity, disparate bunch lengthening circumstances correspond to different parts in the area, color bar presents the final bunch length from $1 \mathrm{~cm}$ to $6 \mathrm{~cm}$. In which cool color area presents the near optimal lengthening condition, while the warm color area indicates that the bunch is overstretched.
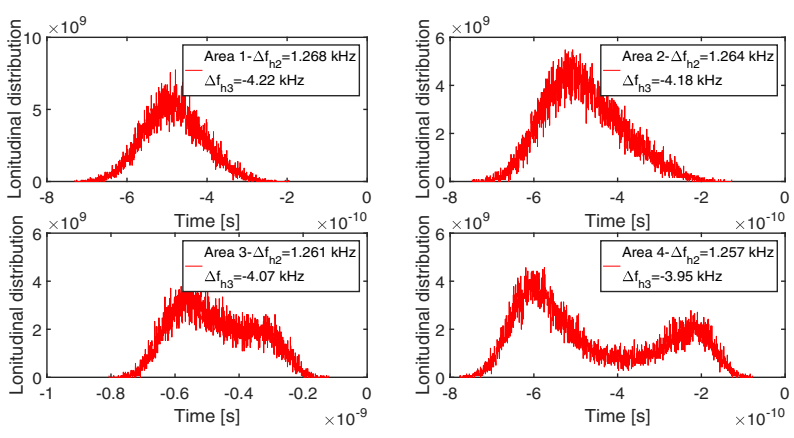

FIG. 10. Final distribution varies with the detuning frequencies of the 2nd and 3rd harmonic cavities, the bunch is nearly optimal lengthened in the upper two figures, while the bunch is overstretched in the bottom figures.

and the impedances of other elements in the ring are also neglected, as well as the intrabeam scattering. All these effects are needed to perform one self-consistent simulation, and this part is still under study.

\section{CONSIDERING THE ENERGY LOSS PER TURN RELATED TO THE MOMENTUM DEVIATION}

The greatest challenge in a practical project using such longitudinal injection scheme is the design of the ultrafast pulse kicker. Not only a rigorous raise time, but also a nonredundant pulse, otherwise it will have fatal influence on the circulating bunches. In fact, this restriction is able to be relieved when considering the energy loss per turn related to the momentum deviation, in which can be represented as [7]

$$
U_{\delta}=U_{0}(1+\delta)^{3},
$$

where $U_{\delta}$ is the energy loss per turn for the particles with the momentum deviation $\delta, U_{0}$ is the energy loss per turn for the nominal beam energy.
Noted that the formula is not self-consistent for the storage rings with combined function bending magnets. The valid approach is to integrate the instantaneous power $P_{\gamma}=\frac{c C_{\gamma}}{2 \pi} \frac{E^{4}}{\rho^{2}}$ at all radiation elements, where $C_{\gamma}=8.85 \times$ $10^{-5} \mathrm{~m} /(\mathrm{GeV})^{3}, \rho$ is the local radius of curvature. We utilize Eq. (11) just for the preliminary numerical analysis.

Since the longitudinal motion can be described as the following differential equations:

$$
\begin{aligned}
& \frac{d z}{d t}=-c \alpha_{c} \delta, \\
& \frac{d \delta}{d t}=\frac{1}{E_{0} T_{0}}\left[e V(z)-U_{0}(1+\delta)^{3}\right] .
\end{aligned}
$$

Where $c$ is the speed of light, $\alpha_{c}$ is the momentum compaction factor of the storage ring, $\delta$ is the relative momentum deviation. Under the triple-frequency rf system,

$$
\begin{aligned}
V(z)= & V_{f} \sin \left(\omega_{f} \frac{z}{c}+\phi_{f}\right)+V_{h_{1}} \sin \left(\omega_{h_{1}} \frac{z}{c} h_{1}+\phi_{h_{1}}\right) \\
& +V_{h_{2}} \sin \left(\omega_{h_{2}} \frac{z}{c} h_{2}+\phi_{h_{2}}\right),
\end{aligned}
$$

where $\omega_{f}, \omega_{h_{1}}, \omega_{h_{2}}$ are the angular frequency of the triplefrequency rf system respectively. Naturally the longitudinal motion including the energy-dependent synchrotron radiation loss can be derived by solving the above differential equations.

Figure 11 presents the corresponding results, the lines in disparate color stand for the longitudinal motion in a different energy deviation with respect to the nominal energy, the black star and black hexagram are the same with Fig. 3. The black imaginary line stands for the momentum deviation 3\%, which is our bucket height.

The longitudinal acceptance including energy-dependent synchrotron radiation loss will be similar with the shape of a "golf club," just like in Fig. 11, and its shaft extends towards the neighboring bucket. The particles outside the original static bucket are able to be captured within such

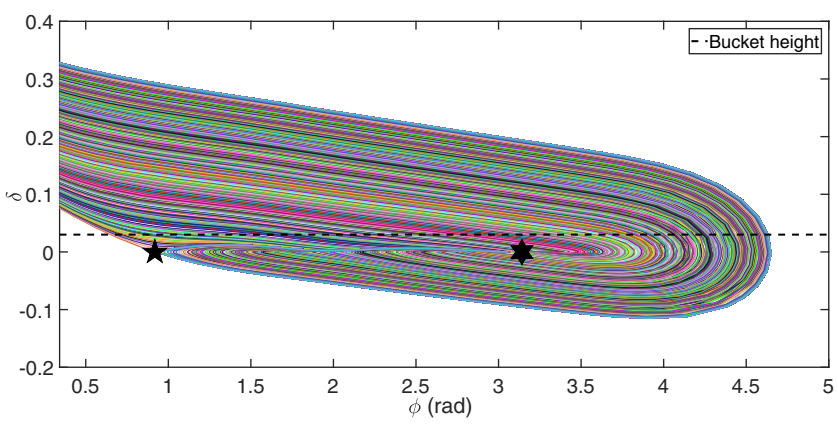

FIG. 11. Longitudinal acceptance including energy-dependent synchrotron radiation loss, the lines in the disparate color stand for the longitudinal motion in different energy deviation with respect to the circulating bunch, and the black star and black hexagram are the same with Fig. 3. 


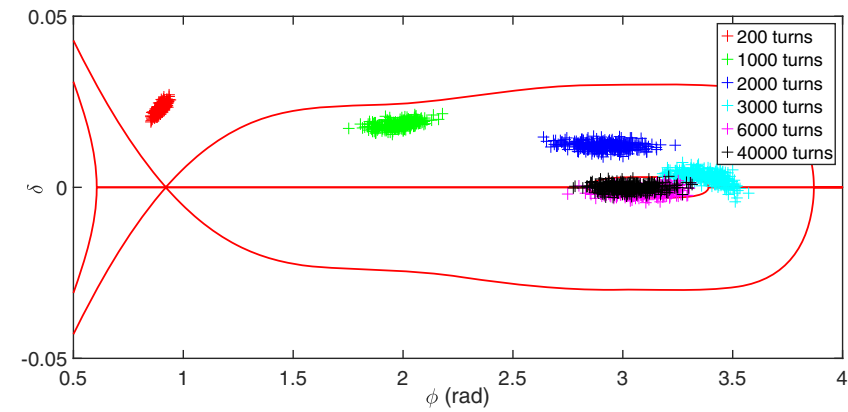

FIG. 12. Particle distribution in the longitudinal plane, in which the beam is injected at $\mathrm{dt}=-2.3 \mathrm{~ns}, \mathrm{dp}=0.03$, and it can be merged to the center of the main bucket.

new longitudinal acceptance. The bunch from the injector with higher momentum deviation can be injected at further longitudinal point. And it will still merge to the center of the main bucket including the synchrotron radiation damping effect.

Figure 12 presents the similar injection process where the bunch is injected at $\mathrm{dt}=-2.3 \mathrm{~ns}, \mathrm{dp}=0.03$, noted that our original injection point is at $\mathrm{dt}=-2 \mathrm{~ns}, \mathrm{dp}=0$ in the above section. The bunch can merge to the center of the bucket without any particle loss.

It needs to be emphasized that there is just bare lattice in our simulation, particles may get lost when we put random errors to the magnets, since such process is connected with the local momentum acceptance (LMA) when there is momentum deviation of the injected bunch. And it may reduce the injection efficiency if there are particles get lost initially. All in all this is still worth trying this approach to relief the pressure to the ultrafast pulse kicker.

\section{CONCLUSION}

Till now we present a preliminary but novel longitudinal injection scheme based on a triple-frequency rf system for future light sources. Utilizing such delicately designed $\mathrm{rf}$ system, we can construct a commodious main bucket which satisfies our injection requirements. Without any extra manual adjustments to the rf parameters, which can be decided from the initial design and remain unchanged during all the operation period. The qualified ultrafast kickers are still required in our design, particularly the raise time needs to be insured within 2 ns. Various lattices of HEPS have been adopted in our incipient study, as our complete mathematical analysis do not decide on any particularities of the lattice, the scheme do have versatility to the future light sources, in spite of we just present the results to one particular lattice in this paper.

Relevant simulation studies we had done are just preliminary, in which more error results and different filling pattern in the storage ring need to be explored in future work, as well as various instabilities.

\section{ACKNOWLEDGMENTS}

We wish to acknowledge the support of Michael Borland, who provided fruitful and patient guidance on the usage of ELEGANT. And we also thank Zhe Duan and Tim Berenc for the abundant helpful encouragements and suggestions.

\section{APPENDIX: A FEASIBLE APPROACH TO SEARCH FOR THE RF PARAMETERS}

Since the first step to construct such complex rf system is to derive all the $\mathrm{rf}$ parameters satisfying the constraints described in Sec. III, here are all seven variables $V_{f}$, $V_{h_{1}}, V_{h_{2}}, \phi_{f}, \phi_{h_{1}}, \phi_{h_{2}}$ and $\phi_{b}$. For easier writing, denoting $\phi_{1}=\phi_{f}, \phi_{2}=\phi_{h_{1}}, \phi_{3}=\phi_{h_{2}}, V_{1}=V_{f}, V_{2}=V_{h_{1}}, V_{3}=V_{h_{2}}$.

In this section we will offer a feasible approach to reduce the number of variables by Eq. (4)-Eq. (6) in Sec. III, and display the final analytical results. First we rewrite these equations described in Sec. III:

$$
\left\{\begin{array}{l}
P\left(\phi_{s}\right)=0 \\
P^{\prime}\left(\phi_{s}\right)=0 \\
P\left(\phi_{b}\right)=P_{\max } \\
P^{\prime}\left(\phi_{b}\right)=0 \\
P^{\prime \prime}\left(\phi_{s}\right)=0
\end{array}\right.
$$

Since the first equation $P\left(\phi_{s}\right)=0$ is satisfied automatically by the definition to the potential energy function,

$$
\begin{aligned}
P(\phi ; t)= & U_{0}\left(\phi-\phi_{s}\right)+V_{1}\left[\cos \left(\phi+\phi_{1}\right)-\cos \left(\phi_{s}+\phi_{1}\right)\right] \\
& +\frac{V_{2}}{2}\left[\cos \left(2 \phi+\phi_{2}\right)-\cos \left(2 \phi_{s}+\phi_{2}\right)\right] \\
& +\frac{V_{3}}{3}\left[\cos \left(3 \phi+\phi_{3}\right)-\cos \left(3 \phi_{s}+\phi_{3}\right)\right] . \quad(\mathrm{A} 2)
\end{aligned}
$$

The surplus four equations can be combined into a system of nonlinear equations,

$$
\left\{\begin{array}{l}
-V_{1} \cos \left(\phi_{1}+\phi_{s}\right)-2 V_{2} \cos \left(2 \phi_{s}+\phi_{2}\right)-3 V_{3} \cos \left(3 \phi_{s}+\phi_{3}\right)=0, \\
U_{0}-V_{1} \sin \left(\phi_{1}+\phi_{s}\right)-V_{2} \sin \left(2 \phi_{s}+\phi_{2}\right)-V_{3} \sin \left(3 \phi_{s}+\phi_{3}\right)=0, \\
U_{0}-V_{1} \sin \left(\phi_{b}+\phi_{1}\right)-V_{2} \sin \left(2 \phi_{b}+\phi_{2}\right)-V_{3} \sin \left(3 \phi_{b}+\phi_{3}\right)=0, \\
U_{0}\left(\phi_{b}-\phi_{s}\right)+V_{1}\left[\cos \left(\phi_{b}+\phi_{1}\right)-\cos \left(\phi_{s}+\phi_{1}\right)\right]+ \\
\frac{V_{2}}{2}\left[\cos \left(2 \phi_{b}+\phi_{2}\right)-\cos \left(2 \phi_{s}+\phi_{2}\right)\right]+\frac{V_{3}}{3}\left[\cos \left(3 \phi_{b}+\phi_{3}\right)-\cos \left(3 \phi_{s}+\phi_{3}\right)\right]=P_{\max } .
\end{array}\right.
$$


One can find that the number of independent variables can be reduced from seven to three by solving the above nonlinear equations. In our analysis, we choose the initial independent variables are $\phi_{1}, \phi_{2}, \phi_{b}$, then we just have to solve the other variables $V_{1}, V_{2}, V_{3}, \phi_{3}$ expressed by these three independent variables.
Then we handle with the first three equations in Eq. (A3) to solve $V_{1}, V_{2}, V_{3}$ as the functions of all the phases $\phi_{1}, \phi_{2}, \phi_{3}, \phi_{b}$. In this step one can find that it is indeed a linear problem, as we do not have to unfold any sine functions or cosine functions. It can be done by Mathematica or other softwares, here we present our results,

$$
\begin{aligned}
V_{1}\left(\phi_{1}, \phi_{2}, \phi_{3}, \phi_{b}\right)= & {\left[3 U_{0} \cos \left(\phi_{3}+3 \phi_{s}\right) \sin \left(\phi_{2}+2 \phi_{b}\right)-2 U_{0} \cos \left(\phi_{2}+2 \phi_{s}\right) \sin \left(\phi_{3}+3 \phi_{b}\right)-3 U_{0} \cos \left(\phi_{3}+3 \phi_{s}\right)\right.} \\
& \left.\sin \left(\phi_{2}+2 \phi_{s}\right)+2 U_{0} \cos \left(\phi_{2}+2 \phi_{s}\right) \sin \left(\phi_{3}+3 \phi_{s}\right)\right] /\left[3 \cos \left(\phi_{3}+3 \phi_{s}\right) \sin \left(\phi_{2}+2 \phi_{b}\right)\right. \\
& \sin \left(\phi_{1}+\phi_{s}\right)-2 \cos \left(\phi_{2}+2 \phi_{s}\right) \sin \left(\phi_{3}+3 \phi_{b}\right) \sin \left(\phi_{1}+\phi_{s}\right)-3 \cos \left(\phi_{3}+3 \phi_{s}\right) \sin \left(\phi_{1}+\phi_{b}\right) \\
& \sin \left(\phi_{2}+2 \phi_{s}\right)+\cos \left(\phi_{1}+\phi_{s}\right) \sin \left(\phi_{3}+3 \phi_{b}\right) \sin \left(\phi_{2}+2 \phi_{s}\right)+2 \cos \left(\phi_{2}+2 \phi_{s}\right) \sin \left(\phi_{1}+\phi_{b}\right) \\
& \left.\sin \left(\phi_{3}+3 \phi_{s}\right)-\cos \left(\phi_{1}+\phi_{s}\right) \sin \left(\phi_{2}+2 \phi_{b}\right) \sin \left(\phi_{3}+3 \phi_{s}\right)\right],
\end{aligned}
$$

$V_{2}\left(\phi_{1}, \phi_{2}, \phi_{3}, \phi_{b}\right)=\left[U_{0} \sin \left(\phi_{3}+3 \phi_{b}\right)-V_{1}\left(\phi_{1}, \phi_{2}, \phi_{3}, \phi_{b}\right) \sin \left(\phi_{3}+3 \phi_{b}\right) \sin \left(\phi_{1}+\phi_{s}\right)-U_{0} \sin \left(\phi_{3}+3 \phi_{s}\right)\right.$

$$
\left.+V_{1}\left(\phi_{1}, \phi_{2}, \phi_{3}, \phi_{b}\right) \sin \left(\phi_{1}+\phi_{b}\right) \sin \left(\phi_{3}+3 \phi_{s}\right)\right] /\left[\sin \left(\phi_{3}+3 \phi_{b}\right) \sin \left(\phi_{2}+2 \phi_{s}\right)\right.
$$$$
\left.-\sin \left(\phi_{2}+2 \phi_{b}\right) \sin \left(\phi_{3}+3 \phi_{s}\right)\right] \text {, }
$$

$V_{3}\left(\phi_{1}, \phi_{2}, \phi_{3}, \phi_{b}\right)=\csc \left(\phi_{3}+3 \phi_{b}\right)\left[U_{0}-V_{1}\left(\phi_{1}, \phi_{2}, \phi_{3}, \phi_{b}\right) \sin \left(\phi_{1}+\phi_{b}\right)-V_{2}\left(\phi_{1}, \phi_{2}, \phi_{3}, \phi_{b}\right) \sin \left(\phi_{2}+2 \phi_{b}\right)\right]$.

Till now we obtain the functions $V_{1}\left(\phi_{1}, \phi_{2}, \phi_{3}, \phi_{b}\right), V_{2}\left(\phi_{1}, \phi_{2}, \phi_{3}, \phi_{b}\right), V_{3}\left(\phi_{1}, \phi_{2}, \phi_{3}, \phi_{b}\right)$, then substituting these three expressions into the last equation in Eq. (A3), and solving the independent variable $\phi_{3}$. One can make polynomial expansion and extract all the terms related with $\cos \left(\phi_{3}\right)$ and $\sin \left(\phi_{3}\right)$, here are the final tedious results,

$$
\begin{aligned}
\cos \phi_{3}\left(\phi_{1}, \phi_{2}, \phi_{b}\right)= & 18\left(P_{\max }-U_{0} \phi_{b}+U_{0} \phi_{s}\right) \cos \left(\phi_{1}+\phi_{2}\right)-18\left(P_{\max }-U_{0} \phi_{b}+U_{0} \phi_{s}\right) \cos \left(\phi_{1}-\phi_{2}+2 \phi_{b}\right) \\
& +6\left(P_{\max }-U_{0} \phi_{b}+U_{0} \phi_{s}\right) \cos \left(\phi_{1}-\phi_{2}-4 \phi_{s}\right)+12\left(P_{\max }-U_{0} \phi_{b}+U_{0} \phi_{s}\right) \cos \left(\phi_{1}-\phi_{2}\right. \\
& \left.-\phi_{b}-3 \phi_{s}\right)+18\left(P_{\max }-U_{0} \phi_{b}+U_{0} \phi_{s}\right) \cos \left(\phi_{1}-\phi_{2}-2 \phi_{b}-2 \phi_{s}\right)+24\left(P_{\max }-U_{0} \phi_{b}+U_{0} \phi_{s}\right) \\
& \cos \left(\phi_{1}+\phi_{2}+\phi_{b}-\phi_{s}\right)+12\left(P_{\max }-U_{0} \phi_{b}+U_{0} \phi_{s}\right) \cos \left(\phi_{1}+\phi_{2}-\phi_{b}+\phi_{s}\right)-36\left(P_{\max }-U_{0}\right. \\
& \left.\phi_{b}+U_{0} \phi_{s}\right) \cos \left(\phi_{1}-\phi_{2}+\phi_{b}+\phi_{s}\right)-24\left(P_{\max }-U_{0} \phi_{b}+U_{0} \phi_{s}\right) \cos \left(\phi_{1}-\phi_{2}+2 \phi_{s}\right) \\
& +6\left(P_{\max }-U_{0} \phi_{b}+U_{0} \phi_{s}\right) \cos \left(\phi_{1}+\phi_{2}-2 \phi_{b}+2 \phi_{s}\right)-12\left(P_{\max }-U_{0} \phi_{b}+U_{0} \phi_{s}\right) \cos \left(\phi_{1}-\phi_{2}\right. \\
& \left.-\phi_{b}+3 \phi_{s}\right)-6\left(P_{\max }-U_{0} \phi_{b}+U_{0} \phi_{s}\right) \cos \left(\phi_{1}+\phi_{2}+2 \phi_{b}+4 \phi_{s}\right)-6 U_{0} \sin \left(\phi_{1}+\phi_{2}\right)-U_{0} \\
& \sin \left(\phi_{1}-\phi_{2}-4 \phi_{b}\right)-13 U_{0} \sin \left(\phi_{1}-\phi_{2}+2 \phi_{b}\right)+11 U_{0} \sin \left(\phi_{1}-\phi_{2}-4 \phi_{s}\right)+10 U_{0} \sin \left(\phi_{1}\right. \\
& \left.-\phi_{2}-\phi_{b}-3 \phi_{s}\right)-18 U_{0} \sin \left(\phi_{1}-\phi_{2}-2 \phi_{b}-2 \phi_{s}\right)+9 U_{0} \sin \left(\phi_{1}+\phi_{2}+2 \phi_{b}-2 \phi_{s}\right)-2 U_{0} \\
& \sin \left(\phi_{1}-\phi_{2}-3 \phi_{b}-\phi_{s}\right)+14 U_{0} \sin \left(\phi_{1}+\phi_{2}+\phi_{b}-\phi_{s}\right)-8 U_{0} \sin \left(\phi_{1}-\phi_{2}+3 \phi_{b}-\phi_{s}\right) \\
& -6 U_{0} \sin \left(\phi_{1}+\phi_{2}-\phi_{b}+\phi_{s}\right)-18 U_{0} \sin \left(\phi_{1}-\phi_{2}+\phi_{b}+\phi_{s}\right)+17 U_{0} \sin \left(\phi_{1}-\phi_{2}+2 \phi_{s}\right)-11 U_{0} \\
& \sin \left(\phi_{1}+\phi_{2}-2 \phi_{b}+2 \phi_{s}\right)+U_{0} \sin \left(\phi_{1}+\phi_{2}+4 \phi_{b}+2 \phi_{s}\right)+22 U_{0} \sin \left(\phi_{1}-\phi_{2}-\phi_{b}+3 \phi_{s}\right) \\
& -6 U_{0} \sin \left(\phi_{1}+\phi_{2}+3 \phi_{b}+3 \phi_{s}\right)+3 U_{0} \sin \left(\phi_{1}+\phi_{2}+2 \phi_{b}+4 \phi_{s}\right)+2 U_{0} \\
& \sin \left(\phi_{1}+\phi_{2}+\phi_{b}+5 \phi_{s}\right),
\end{aligned}
$$

$\sin \phi_{3}\left(\phi_{1}, \phi_{2}, \phi_{b}\right)=6 U_{0} \cos \left(\phi_{1}+\phi_{2}\right)+U_{0} \cos \left(\phi_{1}-\phi_{2}-4 \phi_{b}\right)-13 U_{0} \cos \left(\phi_{1}-\phi_{2}+2 \phi_{b}\right)-11 U_{0} \cos \left(\phi_{1}-\phi_{2}\right.$

$\left.-4 \phi_{s}\right)-10 U_{0} \cos \left(\phi_{1}-\phi_{2}-\phi_{b}-3 \phi_{s}\right)+18 U_{0} \cos \left(\phi_{1}-\phi_{2}-2 \phi_{b}-2 \phi_{s}\right)-9 U_{0} \cos \left(\phi_{1}+\phi_{2}\right.$

$\left.+2 \phi_{b}-2 \phi_{s}\right)+2 U_{0} \cos \left(\phi_{1}-\phi_{2}-3 \phi_{b}-\phi_{s}\right)-14 U_{0} \cos \left(\phi_{1}+\phi_{2}+\phi_{b}-\phi_{s}\right)-8 U_{0} \cos \left(\phi_{1}\right.$

$\left.-\phi_{2}+3 \phi_{b}-\phi_{s}\right)+6 U_{0} \cos \left(\phi_{1}+\phi_{2}-\phi_{b}+\phi_{s}\right)-18 U_{0} \cos \left(\phi_{1}-\phi_{2}+\phi_{b}+\phi_{s}\right)+17 U_{0} \cos \left(\phi_{1}\right.$ 


$$
\begin{aligned}
& \left.-\phi_{2}+2 \phi_{s}\right)+11 U_{0} \cos \left(\phi_{1}+\phi_{2}-2 \phi_{b}+2 \phi_{s}\right)+U_{0} \cos \left(\phi_{1}+\phi_{2}+4 \phi_{b}+2 \phi_{s}\right)+22 U_{0} \cos \left(\phi_{1}\right. \\
& \left.-\phi_{2}-\phi_{b}+3 \phi_{s}\right)-6 U_{0} \cos \left(\phi_{1}+\phi_{2}+3 \phi_{b}+3 \phi_{s}\right)+3 U_{0} \cos \left(\phi_{1}+\phi_{2}+2 \phi_{b}+4 \phi_{s}\right)+2 U_{0} \\
& \cos \left(\phi_{1}+\phi_{2}+\phi_{b}+5 \phi_{s}\right)+18\left(P_{\max }-U_{0} \phi_{b}+U_{0} \phi_{s}\right) \sin \left(\phi_{1}+\phi_{2}\right)+18\left(P_{\max }-U_{0} \phi_{b}+U_{0} \phi_{s}\right) \\
& \sin \left(\phi_{1}-\phi_{2}+2 \phi_{b}\right)+6\left(P_{\max }-U_{0} \phi_{b}+U_{0} \phi_{s}\right) \sin \left(\phi_{1}-\phi_{2}-4 \phi_{s}\right)+12\left(P_{\max }-U_{0} \phi_{b}+U_{0} \phi_{s}\right) \\
& \sin \left(\phi_{1}-\phi_{2}-\phi_{b}-3 \phi_{s}\right)+18\left(P_{\max }-U_{0} \phi_{b}+U_{0} \phi_{s}\right) \sin \left(\phi_{1}-\phi_{2}-2 \phi_{b}-2 \phi_{s}\right)+24\left(P_{\max }-U_{0}\right. \\
& \left.\phi_{b}+U_{0} \phi_{s}\right) \sin \left(\phi_{1}+\phi_{2}+\phi_{b}-\phi_{s}\right)+12\left(P_{\max }-U_{0} \phi_{b}+U_{0} \phi_{s}\right) \sin \left(\phi_{1}+\phi_{2}-\phi_{b}+\phi_{s}\right) \\
& +36\left(P_{\max }-U_{0} \phi_{b}+U_{0} \phi_{s}\right) \sin \left(\phi_{1}-\phi_{2}+\phi_{b}+\phi_{s}\right)+24\left(P_{\max }-U_{0} \phi_{b}+U_{0} \phi_{s}\right) \sin \left(\phi_{1}-\phi_{2}\right. \\
& \left.+2 \phi_{s}\right)+6\left(P_{\max }-U_{0} \phi_{b}+U_{0} \phi_{s}\right) \sin \left(\phi_{1}+\phi_{2}-2 \phi_{b}+2 \phi_{s}\right)+12\left(P_{\max }-U_{0} \phi_{b}+U_{0} \phi_{s}\right) \\
& \sin \left(\phi_{1}-\phi_{2}-\phi_{b}+3 \phi_{s}\right)+6\left(P_{\max }-U_{0} \phi_{b}+U_{0} \phi_{s}\right) \sin \left(\phi_{1}+\phi_{2}+2 \phi_{b}+4 \phi_{s}\right) .
\end{aligned}
$$

At last we obtain the function $\phi_{3}$ using the independent variables $\phi_{1}, \phi_{2}, \phi_{b}$, and we can substitute it back into the Eq. (A4)-Eq. (A6) to derive all the other variables.

To search the appropriate if parameters, the following steps are recommended, (i) Give the random values of $\phi_{1}, \phi_{2}, \phi_{b}$, (ii) Calculate if phase $\phi_{3}$ by Eq. (A7)Eq. (A8), (iii) Calculate rf voltages $V_{1}, V_{2}, V_{3}$ by Eq. (A4)Eq. (A6), (iv) Make rational judgements to this set of $\mathrm{rf}$ parameters, using the potential energy function $P(\phi ; t)$ and the longitudinal acceptance conducted by them, and ascertain whether they are satisfied with the requirements. One can handle the above process within a simple nested loop up to three layers, and till there are good results.

[1] R. Hettel, DLSR design and plans: an international overview, Synchrotron Radiat. News 21, 843 (2014).

[2] D. Einfeld, M. Plesko, and J. Schaper, First multi-bend achromat lattice consideration, Synchrotron Radiat. News 21, 856 (2014).

[3] C. Gough and M. Mailand, in Proceedings of the 19th Particle Accelerator Conference, Chicago, IL, 2001 (IEEE, Piscataway, 2001), p. 3741.

[4] H. Takaki, N. Nakamura, Y. Kobayashi, K. Harada, T. Miyajima, A. Ueda, S. Nagahashi, M. Shimada, T. Obina, and T. Honda, Beam injection with a pulsed sextupole magnet in an electron storage ring, Phys. Rev. ST Accel. Beams 13, 020705 (2010).

[5] K. Harada, Y. Kobayashi, T. Miyajima, and S. Nagahashi, New injection scheme using a pulsed quadrupole magnet in electron storage rings, Phys. Rev. ST Accel. Beams 10, 123501 (2007).

[6] S. C. Leemann, Pulsed sextupole injection for Sweden's new light source MAX IV, Phys. Rev. ST Accel. Beams 15, 050705 (2012).

[7] M. Aiba, M. Böge, F. Marcellini, Á. S. Hernández, and A. Streun, Longitudinal injection scheme using short pulse kicker for small aperture electron storage rings, Phys. Rev. ST Accel. Beams 18, 020701 (2015).
[8] M. Borland, A super-bright storage ring alternative to an energy recovery linac, Nucl. Instrum. Methods Phys. Res., Sect. A 557, 230 (2006).

[9] A. Xiao, M. Borland, and C. Yao, in Proceedings of the 25th Particle Accelerator Conference, PAC-2013, Pasadena, CA, 2013 (IEEE, New York, 2013), p. 1076.

[10] C. Steier, A. Anders, T. Luo, T. Oliver, C. Pappas, S. D. Santis, C. Sun, C. Swenson, and W. Waldron, in Proceedings of the 8th International Particle Accelerator Conference, IPAC-2017, Copenhagen, Denmark (IEEE, New York, 2017), p. 2821.

[11] G. N. Kulipanov, in Proceedings of the 8th International Conference on High-Energy Accelerators, Geneva, Switzerland (European Organization for Nuclear Research, Geneva, 1971) p. 138.

[12] B. C. Jiang, Z.T. Zhao, S. Q. Tian, M. Z. Zhang, and Q. L. Zhang, Using a double-frequency RF system to facilitate on-axis beam accumulation in a storage ring, Nucl. Instrum. Methods Phys. Res., Sect. A 814, 1 (2016).

[13] G. Xu, J. Chen, Z. Duan, and J. Qiu, in Proceedings of the 7th International Particle Accelerator Conference, IPAC2016, Busan, Korea (IEEE, New York, 2016), p. 2032.

[14] M.-A. Tordéux, in Topical Workshop on Injection and Injection System, Berlin, Germany, 2017, unpublished.

[15] G. Xu, X. H. Cui, and Z. Duan, in Proceedings of the 9th International Particle Accelerator Conference, IPAC2018, Vancouver, BC, Canada (IEEE, New York, 2018), pp. 1375-1378.

[16] B. I. Grishanov, F. V. Podgorny, J. Rümmler, and V. D. Shiltsev, Very fast kicker with high repetition rate for accelerator applications, Nucl. Instrum. Methods Phys. Res., Sect. A 396, 28 (1997).

[17] J. H. Chen, H. Shi, L. Wang, Z. Duan, N. Wang, L. H. Huo, G. W. Wang, X. L. Shi, and P. Liu, in Proceedings of the 9th International Particle Accelerator Conference, IPAC-2018, Vancouver, BC, Canada (IEEE, New York, 2018), pp. 2846-2849.

[18] M. Borland, Elegant: A flexible SDDS-compliant code for accelerator simulation, Advanced Photon Source Technical Report No. LS-287, 2000.

[19] T. Berenc and M. Borland, in Proceedings of the 6th International Particle Accelerator Conference, IPAC-2015, Richmond, VA, USA (IEEE, New York, 2015), pp. 540-542. 
[20] P. F. Tavares, A. Andersson, A. Hansson, and J. Breunlin, Equilibrium bunch density distribution with passive harmonic cavities in a storage ring, Phys. Rev. ST Accel. Beams 17, 064401 (2014).

[21] J. M. Byrd and M. Georgsson, Lifetime increase using passive harmonic cavities in synchrotron light sources, Phys. Rev. ST Accel. Beams 4, 030701 (2001).
[22] J. M. Byrd, S. D. Santis, J. Jacob, and V. Serriere, Transient beam loading effects in harmonic rf systems for light sources, Phys. Rev. ST Accel. Beams 5, 092001 (2002).

[23] M. Borland, T. Berenc, R. Lindberg, and A. Xiao, in Proceedings of the 6th International Particle Accelerator Conference, IPAC-2015, Richmond, VA, USA (IEEE, New York, 2015), p. 543. 